\title{
MicroscopyEducation
}

\section{Factors to Consider When Selecting Student Microscopes for Schools and Universities}

\section{Vince Vaccarelli}

Leica Microsystems Inc. | 1700 Leider Lane, Buffalo Grove, IL, 60089

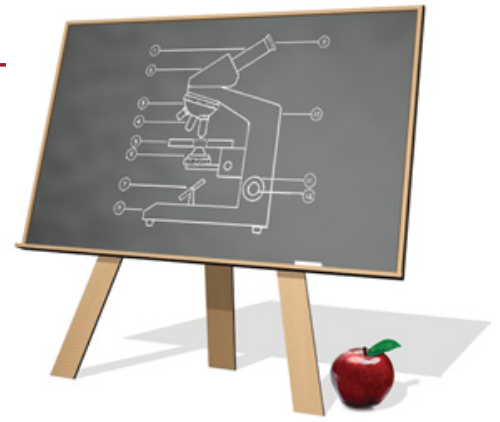

vince.vaccarelli@leica-microsystems.com

\begin{abstract}
Selection of microscopes for student use involves many factors. The microscopes must stand up to daily use by students with little experience, be constantly up and running, and fit the budget requirements. Especially with student microscopes, practical aspects can be important. Thus, size, weight, cabling, and design should be taken into consideration even before the microscope and its accessories enter the decision process. If chosen carefully, educational microscopes can delight young minds in schools and universities, and, ideally, the experience will fascinate them enough that they decide to make science their profession.
\end{abstract}

Keywords: Student microscopes, durability, usability, resolution, accessories

\section{Introduction}

Selecting educational microscopes is not an easy task for teachers. These microscopes must stand up to daily student use, be constantly up and running, and often fit strict budget requirements. Especially with student microscopes, practical aspects can play a significant role. Thus, their size, weight, cabling, and design are important for their daily use, and these factors should be taken into account before considering the purchase of microscopes and their accessories. If chosen carefully, educational microscopes can open windows to a cosmos of minute detail that can delight young minds in schools and universities and fascinate them enough so that they consider a career in science. This article describes various factors to consider when selecting microscopes for educational settings.

\section{Classroom Environment}

From our experience, there are two types of classrooms. The first is the laboratory. Microscopes are set up once and remain at the same place all the time (Figure 1). There may be long rows of tables, often with little space between the rows. In this case, fewer cables around the microscope will create a better workspace. Microscopes could become dislodged if students' arms or bags get caught in cables when they take their seats.
The second type of classroom has storage space for microscopes (Figure 2). The microscopes are taken out of storage, set up on the worktops at the beginning of class, and put away again at the end. In this case, there are several factors to consider.

Size. Do the microscopes fit the storage space? There should be enough space between the instruments and perhaps dividers, so that they do not touch. It also makes sense to check that moveable parts are separated enough so that they are not easily damaged.

Weight. Because the microscopes are moved for every lesson, they should be easy for one person to carry. Also take a look at the design. It may be challenging to grasp a microscope securely if it does not have a handle.

Cables. If cables hang down from the microscopes, they can tangle in storage as well as on the way to the benches. Imagine thirty students carrying microscopes and dragging the plugs behind them on the floor, creating tripping hazards.

For both labs and classrooms with storage capacity, the environmental conditions are worth considering. If 


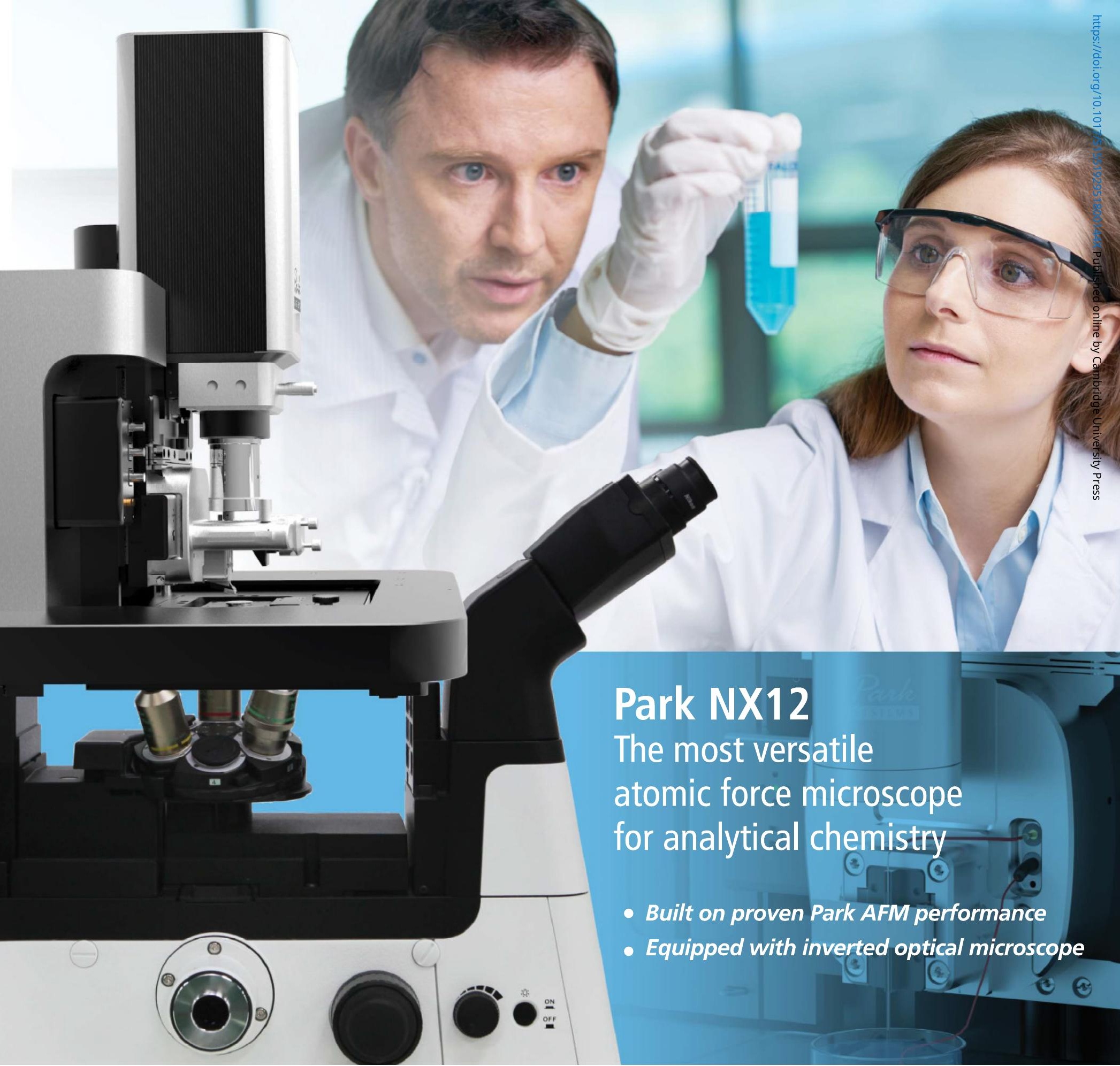

Proven Performance

The Park NX12 is based on the Park NX10, one of the most trusted and widely used AFMs for research. Users can rest assured that they are taking measurements with a cutting-edge tool.
Built for Versatility

Multi-user labs need a versatile microscope to meet a wide range of needs. The Park NX12 was built from the ground up to be a flexible modular platform to allow shared facilities to invest in a single AFM to perform any task.
Competitive Pricing

Early career researchers need to do great work with cost-effective tools. Despite its outstanding pedigree, the Park NX12 is priced affordably_ideal for those on a constrained budget.

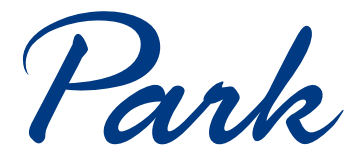

S Y S TE M S parksystems.com 


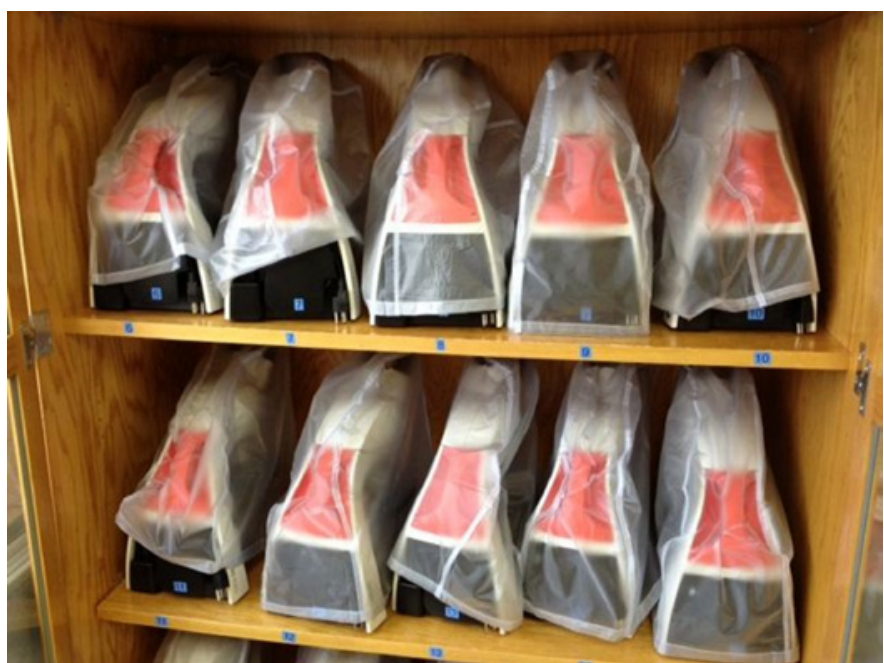

Figure 2: Depository shelves with student microscope stands at Erie Community College. Courtesy of Mary Anne Silsby, Erie Community College, Buffalo, NY.

temperatures and humidity in the rooms change, specific coatings for microscope parts may prevent the growth of fungus and bacteria.

\section{Microscope Construction}

Student microscopes go through many hands every day. Since they will be used for many years, they should be constructed to last. Thus, the design and construction should be examined carefully.

Maintenance. Servicing the microscopes must be easy. Educational microscopes must be easy to clean and maintain. When needed, an on-site service call should be available.

Robustness. Microscopes should not be delicate in construction. Their bases should be planted firmly on the top of the workspace. They also should be well balanced and not wobble. In addition, they should have as few removable parts as possible to ensure the next student still has all the microscope parts at his or her disposal.

Durability. It's worth taking a look at the materials the microscopes are manufactured from. The longer microscopes show little wear and tear, the longer students will treat them with more care. Another scratch on a scratched surface matters less than the first scratch. A durable finish can be helpful.

Smoothness. Moveable parts should move smoothly without requiring force.

Labeling. Well-labeled microscope parts help students handle the microscopes properly as well as locate and use with confidence the few movable parts.

\section{Having Fun Learning and Teaching}

Students learn best if they have fun doing so. Microscopy classes have great potential to fascinate children and young adults because the world they see through the oculars is very different from what they see around them.

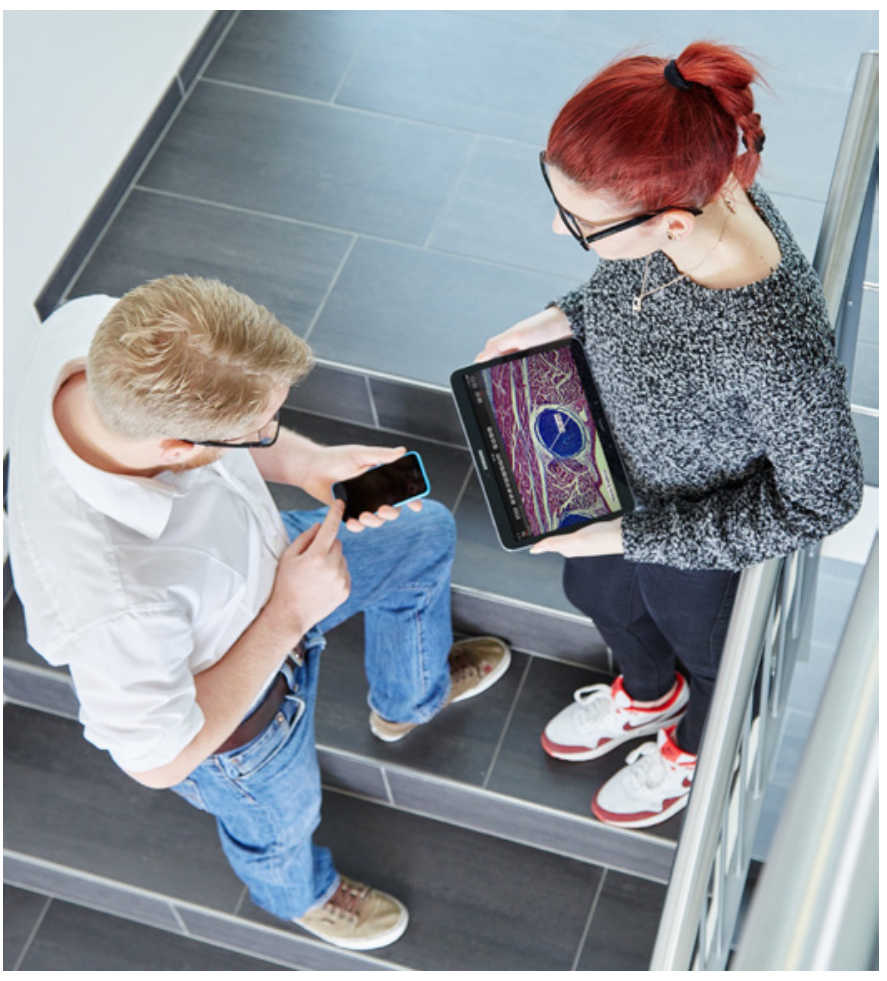

Figure 3: Some cameras create their own Wi-Fi hotspot so that students can access images with their mobile devices or laptops.

Discovering this microcosm is an adventure. Two factors are crucial in promoting the fun factor:

Usability. With only a few adjustments to the microscope, students must be able to see something. This step is important, so that they will remain engaged and teachers will spend less time helping students adjust their instruments. Good labeling and few removable parts make these instruments easy to use.

Great images. The overall optical performance, including the camera, is key to producing images with good contrast, resolution, and color. Good image quality enables students to discover a new world and stay interested.

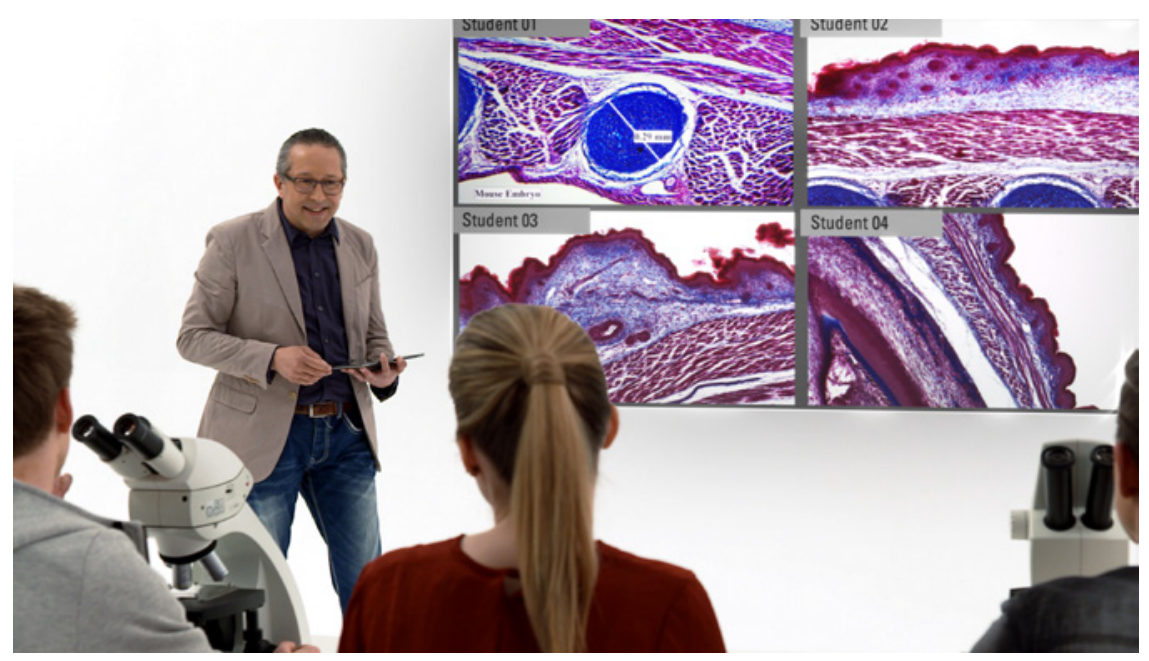

Figure 4: Student microscopes with diverse capabilities can suit different course levels of students. 


\section{Microscope Performance and Accessories}

The choice of microscope also depends on the subject matter and level of the course. In many cases appropriate accessories are needed to perform certain experiments. Microscopes can be equipped to match the requirements of the subject under study.

Accessories. Some accessories depend on the application, for example polarization kits are important for the study of minerals in geology classes. Other accessories can be generally beneficial, for example LED illumination, which lasts much longer than halogen bulbs. The long lifetime of LED lighting eliminates bulb changes and increases microscope uptime. Digital cameras and associated software have become more important in the past few years, as microscopy itself has become more digital. Some cameras can even create their own Wi-Fi hotspot so that students can access images with their mobile devices or laptops (Figures 3).

Course level. Microscopes for biology classes in high school do not need the same capabilities as microscopes for advanced university pathology courses. There are different types of student microscope that can usually match the needs of students (Figure 4) [1].

Budget. The price-performance ratio always requires careful consideration. In the years we have spent talking to teachers, we have learned that nothing is more frustrating for students than lousy images that don't mean anything. For a teacher, nothing is more rewarding than seeing students excited about what they see in the microscope. Therefore, our advice is the following: never compromise on the optical performance. Only great images delight young minds.

\section{Summary}

Answers to the following questions will help you select the microscope that best fits your needs:

1. Where will the microscopes be used and stored? Will they be moved often?

2. How sturdy are the instruments? Will they stand up to daily use? Do the moveable parts move easily for a good user experience?

3. How easily can the microscopes be operated? Will students see something fascinating quickly? This effect should not be underestimated: the easier great images can be seen, the more fun the students will have.

4. For which lessons will the microscope be used? Is any special equipment needed? Which equipment will not be needed?

\section{Acknowledgments}

I would like to thank James DeRose of Leica Microsystems for help with preparing the manuscript.

\section{Reference}

[1] C Müller, "Factors to Consider when Selecting Student Microscopes for Schools and Universities: How studentfriendly equipment makes teachers and students happy." Science Lab (2016) Leica Microsystems. https://www. leica-microsystems.com/science-lab/factors-to-considerwhen-selecting-student-microscopes-for-schools-anduniversities/ (accessed Jan. 7, 2019).

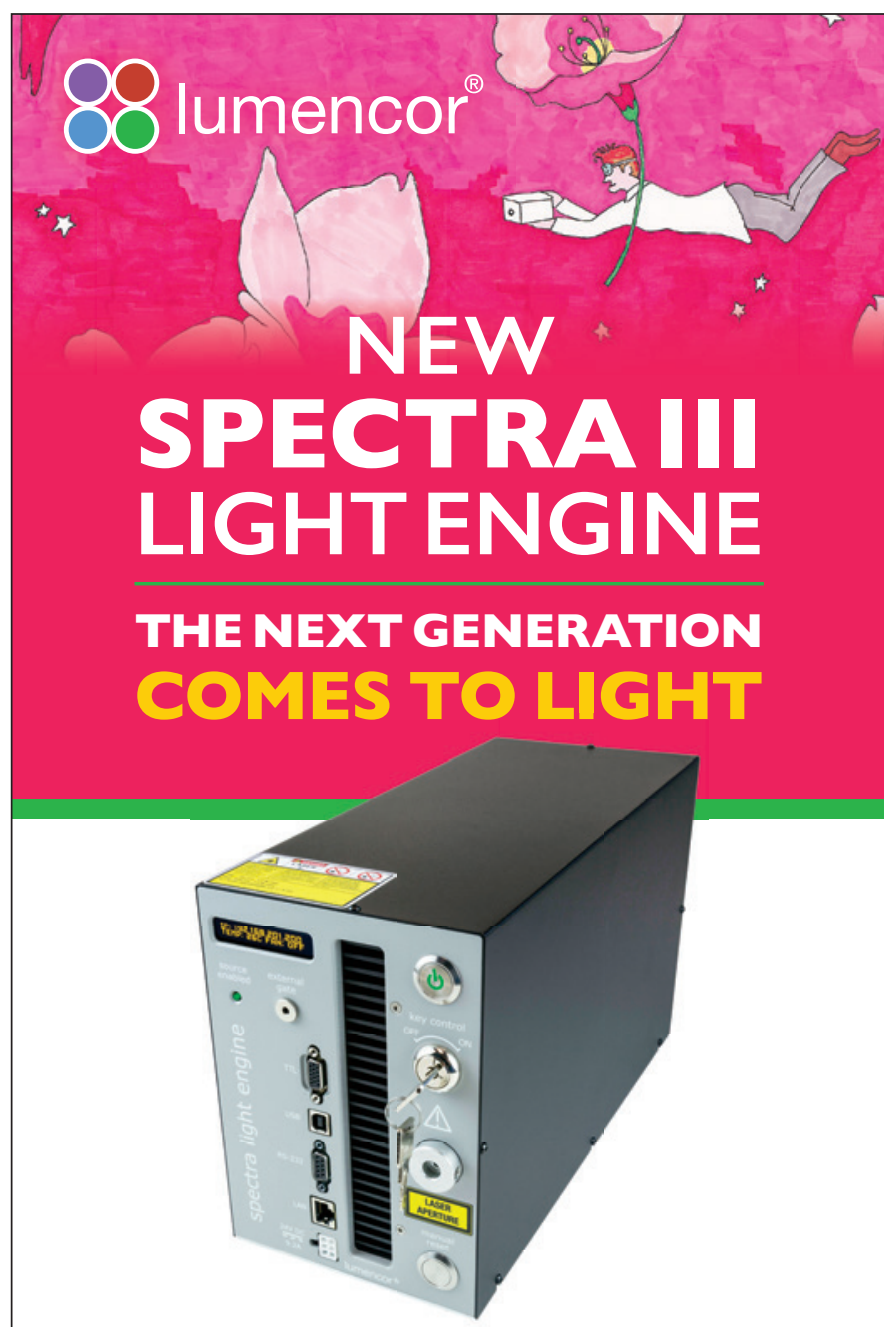

- Breadth: Eight spectrally optimized sources for DAPI, CFP, GFP, YFP, Cy3, mCherry, Cy5, Cy7 excitation

- Power: $\sim 500 \mathrm{~mW} /$ output, $\sim 4 \mathrm{~W}$ total

- Control: Exceptional power and wavelength stability

- Stability: Exceptional reproducibility

- Ideal for quantitation

- Ease of use: Small, cool, pre-aligned, Mercury-Free

- Applications: Fluorescence microscopy among others, OEM customization upon request

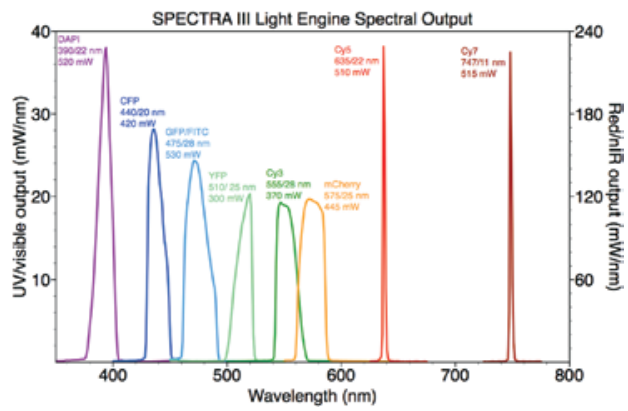

www.lumencor.com 